\title{
Study Protocol on Stroke Management: Role of Nurses and Physiotherapists at the Adult University Teaching Hospital, Lusaka Zambia
}

\author{
Patricia Katowa-Mukwato ${ }^{1^{*}}$, Martha Chalwe Banda ${ }^{2}$, Michael Mumba Kanyanta1, \\ Emmanuel Mwila Musenge1, Peter D.C. Phiri², Victoria Mwiinga-Kalusopa1, Fabian Chapima1, \\ Micah Simpamba², Charity Kapenda2 ${ }^{2}$, Hastings Shula²
}

\author{
${ }^{1}$ Department of Basic and Clinical Nursing Sciences, School of Nursing Sciences, University of Zambia, Lusaka, Zambia \\ ${ }^{2}$ Department of Physiotherapy, School of Health Sciences, University of Zambia, Lusaka, Zambia \\ Email: *patriciakatowamukwato@gmail.com
}

How to cite this paper: Katowa-Mukwato, P., Banda, M.C., Kanyanta, M.M., Musenge, E.M., Phiri, P.D.C., Mwiinga-Kalusopa, V., Chapima, F., Simpamba, M., Kapenda, C. and Shula, H. (2021) Study Protocol on Stroke Management: Role of Nurses and Physiotherapists at the Adult University Teaching Hospital, Lusaka Zambia. Journal of Biosciences and Medicines, 9, 25-37. https://doi.org/10.4236/jbm.2021.99003

Received: June 22, 2021

Accepted: September 4, 2021

Published: September 7, 2021

Copyright $\odot 2021$ by author(s) and Scientific Research Publishing Inc. This work is licensed under the Creative Commons Attribution International License (CC BY 4.0).

http://creativecommons.org/licenses/by/4.0/ (c) (i) Open Access

\begin{abstract}
Background: Management of stroke patients requires a multidisciplinary approach. Literature presents unequivocal evidence of improved outcomes when patients are treated in a stroke unit by multi-disciplinary teams made up of medical doctors, nurses, physiotherapists and occupational therapists. Among the multidisciplinary team members, physiotherapists and nurses are the largest professional workforce working with both stroke patients and survivors. This study, therefore, aims at investigating the role of nurses and physiotherapists in the management of stroke patients at the Adult University Teaching Hospital. Methodology: This study shall utilize a convergent parallel mixed method design where both quantitative and qualitative data will be collected at the same time resulting in two data sets which will eventually be merged at the point of discussion. The quantitative component shall utilize an analytical cross-sectional approach while the qualitative component shall use a qualitative case study approach. The study will be conducted at the Adult University Teaching Hospital in Lusaka. Data will be collected from all the 287 nurses and 37 physiotherapists working in the Emergency Department, Medical Wards, Adult Intensive Care Unit and the Department of Physiotherapy. Quantitative data will be analyzed using Statistical Package for social sciences. Descriptive statistics shall be computed on the role of nurses and physiotherapists in stroke care. In addition, chi-square and fisher's exact tests will be used in the test for associations between variables while binary logistic regression will be used in identifying predictors of the various roles played by nurses and physiotherapists in the management of stroke patients. Qualitative data will be analyzed using thematic analysis in order to generate new concepts and a
\end{abstract}


substantive model of care for stroke patients. Conclusion: Despite research evidence of improved outcomes when stroke patients are managed by a multi-disciplinary team, data on the role of different members of the multidisciplinary care team in stroke management is almost non-existent from the Zambia context. It is therefore hoped that information obtained from this study will be used as a basis for recommending the streamlined role of the nurse and physiotherapist in both acute care and rehabilitation.

\section{Keywords}

Stroke Management, Nursing Role, Physiotherapy Role, Model of Care

\section{Introduction}

This paper reports on a study protocol that was developed to investigate the management of stroke patients by nurses and physiotherapists at the Adult University Teaching Hospital in Lusaka, Zambia. Stroke or Cerebral Vascular Accident (CVA) is the second leading cause of death globally [1] [2]. Stroke accounts for 11 percent of all deaths and represents the largest cause of adult disability. Although patient survival after stroke has improved, it remains a leading cause of severe disability [3]. Traditionally, CVA has been labeled as a disease of the affluent, but reports have shown that Africa which is not affluent, bears a heavy burden of CVA [4]. African countries are undergoing an epidemiological transition driven by socio-demographic and lifestyle changes [4]. With the changing lifestyles, the incidence of CVA is increasing and thus, 28 million people are estimated to die in 10 years' time, just in Africa, due to non-communicable diseases, largely CVA [4] [5]. In Zambia, CVA has been documented to affect the younger population, hypertension being the most common risk factor and the trend is increasing following the advent of human immunodeficiency virus/acquired immunodeficiency syndrome and diabetes mellitus [5].

Stroke care and rehabilitation require a multidisciplinary approach. According to [6], there is unequivocal evidence of improved outcomes when patients are treated in a stroke unit by multi-disciplinary teams. Multidisciplinary teams made up of medical doctors, nurses, physiotherapists and occupational therapists perform stroke rehabilitation in all phases and settings such as hospitals, communities and clinics [7]. Among the multidisciplinary team members, physiotherapists and nurses are the largest professional workforce working with stroke survivors [8]. According to [8], the nursing role in the acute phase of stroke management involves rapid assessment and triage of patients [9], ongoing assessment of vital signs to detect improvement or deterioration and providing or assisting in meeting the respiratory, nutritional, hygiene, elimination and mobility needs of the patient. In addition, Nurses coordinate and accompany the patient's journey of care, liaising with other specialties, facilitating rehabilitation, discharge and follow-up planning, and providing support to patients and families [1]. 
Therefore, in the stroke multidisciplinary care approach, the nurses perform a central role of coordinating other members of the stroke care team [10]. On the other hand, the physiotherapist aims to re-educate movement, sensation and balance in order to enable the patient to reach their potential for recovery of mobility and independence [8]. In addition, Physiotherapists provide rehabilitation services and education for acquired deficits such as motor disability, cognitive impairment, dysphasia, and lower urinary tract symptoms [7].

Despite the significant role played by nurses and physiotherapists in stroke care and rehabilitation as documented in professional literature, literature on the role of the nurses and physiotherapists in acute, sub-acute and post-stroke rehabilitation is in Zambia is sparse. This study, therefore, aims at investigating the nursing and physiotherapy management of stroke patients in Zambia.

\section{Research Questions and Objectives}

\subsection{Research Questions}

1) What is the role of the Nurse and the Physiotherapist in Stroke Management in Zambia?

2) What models of care are used in the Management of stroke patients in Zambia?

\subsection{Research Objectives}

1) To determine the Role of the Nurse and Physiotherapist in the acute, sub-acute and chronic Stroke management

2) To explore the factors influencing Nursing and Physiotherapy Management of Stroke patient

3) To identify Nursing and Physiotherapy Models being used in the management of Stroke Patients.

\section{Material and Methods}

\subsection{Research Design}

This study shall employ a convergent parallel mixed methods design. This design entails collection of both quantitative and qualitative data at the same time resulting in two data sets that will be analyzed separately. Integration of findings from the two data sets will be done at the level of interpretation by comparing quantitative results with qualitative results in the discussion of findings. Use of mixed methods will enable the researchers to draw upon the strength of both quantitative and qualitative approaches in investigating the role of nurses and physiotherapists in stroke management. The quantitative component shall utilize an analytical cross-sectional approach while the qualitative component shall use a qualitative case study approach. The analytical cross-sectional approach will allow for collection of data on the role of the nurse and the physiotherapist in stroke management at one point in time without altering the environment or manipulating the independent variable and to establish relationships among va- 
riables. The use of a qualitative case study will allow the Investigators to get in-depth information regarding the roles of nurses and physiotherapists in managing stroke patients at the Adult University Teaching Hospital (UTH-Adult Hospital) in Lusaka, Zambia.

\subsection{Study Site and Population}

The study will be conducted at the UTH Adult Hospital in Lusaka. The following specific units will be included in the study: The Emergency Department (ED) (Triage Emergency Room, Adult Emergency Medical Unit, Male and Female Admission wards), the Main Intensive Care Unit, Medical wards (EO1 EO2, E11, E12, E21, E22) and Department of Physiotherapy. Clinic 4 shall be taken as the setting for Out Patient care. The UTH Adult Hospital was selected purposively for this study because it is the main referral centre for the entire country with 24 hours emergency services for patients who have acute complaints [11]. The target population shall be nurses and physiotherapists working in medical wards, outpatient departments and the physiotherapy department of the UTH Adult Hospital. The accessible population will include those nurses and physiotherapists that will be present at the study site during the period of the study. Those on leave will not constitute the accessible population.

\subsection{Selection of Participants, Sampling and Sample Size}

\subsubsection{Sample Selection and Sample Size}

A census approach shall be used to recruit participants for quantitative component of the current study. This will imply inclusion of all nurses and physiotherapists in the study setting that will meet the eligibility criteria. Currently there are 287 Nurses working in the selected settings of the UTH-Adult hospital and 37 Physiotherapists. This approach will provide a rich sample for the study that will generate the required information. Participants in the Focus Group Discussions (FDG) shall be selected using purposive sampling method as it will enable selection of participants who will offer valuable insight into the topic under study [12] [13]. The first personnel to be included in the FDG will be the head or the in-charge for the unit who will later suggest other members based on the understanding that they have enough experience and can offer valuable information regarding the management of stroke patients. The selection will ensure that groups are homogeneous to allow for easy of discussion. The number of participants per Focus Group Discussion (FGD) will be $6-12$ as advised by Burns and Grove, [14] and Johnson, and Christensen [15]. A total 4 FDGs will be conducted for nurses dived as (Working in In-Patient Wards, Working in Out Patient Medical Clinic, Working in Emergency Medical Admission and working in the ICU). For Physiotherapist, three FGDs will be conducted as (Working in the ICU, working in patients ward and working with Out Patients).

\subsubsection{Inclusion Criteria}

1) Nurses and physiotherapists working full time in the wards/departments 
selected for the study;

2) Nurses and physiotherapists with a minimum of 6 months working experience;

3) Nurses and physiotherapists that will consent to take part in the study and will meet the other criteria.

\subsubsection{Exclusion Criteria}

Nurses and Physiotherapists who will be working on a part-time basis in selected wards and units will be excluded.

\subsection{Plan for Data Collection, Analysis and Dissemination}

\subsubsection{Data Collection Tools}

The quantitative component shall use two questionnaires, one for nurses and one for physiotherapists. The questionnaires have been adapted from the Stroke foundation clinical guidelines for the stroke management; a quick guide for nursing [16], the Nursing and Midwifery Protocols [17], the Clinical Guidelines on Stroke Rehabilitation [18], and the Clinical Guidelines for Stroke Management [16]. Both Questionnaires will include a section for collection of demographic data in addition to data on the roles regarding stroke care and rehabilitation. For the qualitative component, an interview guides shall be used. The questionnaire will obtain information on the role of nurse and physiotherapist in the management of stroke patients and the models of care they utilize, while the FGD will obtain information on the factors that influence nursing and physiotherapy management of stroke patients.

\subsubsection{Data Collection Technique}

A structured self-report approach will be used for the quantitative component. This will involve use of a structured self-administered questionnaire to report care practices when managing patients with stroke. For qualitative data, Focus Group Discussions will be will be used for collecting detailed information and collective views on stroke care by nurses and physiotherapists. According to Flick [19], group interviews are advantageous in that, they are low cost and rich in data, that they stimulate the respondents and support them in remembering events and that they can lead beyond the answers of a single interviewee. Audio recording of each FGD will be done together with documentation of descriptive field notes. According to [20], descriptive field notes comprise objective descriptions of observed events and conversations to provide information about actions, dialogue, and context of the interview. Documentation of descriptive field notes will facilitate generation of thick descriptions of the data which will enhance transferability [21] assurance of reliability of the data to be collected.

\subsubsection{Data Processing and Analysis}

Quantitative data shall be checked for completeness and entered into version 23 of the Statistical Package for social sciences (SPSS) for analysis. Descriptive statistics shall be computed in the form of frequencies, means and standard devia- 
tions. The data on the dependent variable on the role of the nurse and the physiotherapist in stroke care shall be collected as categorical data. The categories for the dependent variable are yes and no. This will entail use of chi-square tests of associations and fisher exact tests for the bivariate analysis while binary logistic regression will be used to identify predictors of the various roles played by nurses and physiotherapists in the management of Stroke patients. The variables on the roles for the nurses include airway assessment, neurological assessment, assessing level of consciousness, oxygen administration, monitoring bleeding complications, cardiac monitoring, fluid balancing, appropriate positioning, intravenous fluid administration, drug administration, catheter insertion, catheter removal, encouraging elimination, patient turning, range-of-motion exercise, nasogastric tube insertion, and patient discharge. On the other hand, the roles of the physiotherapists include respiratory function assessment, swallowing assessment, muscle tone assessment, body alignment assessment, joint motion assessment, voluntary movement assessment, involuntary activity assessment, sensation assessment, visual-spatial awareness, undesirable compensatory activities, coordination and balance, muscle function, neurological assessment, breathing exercises, appropriate compensatory activities, shaker therapy, thermal tactile stimulation, electrical therapy, progressive resistance exercises, electrical stimulation, sensory specific training, cueing of cadence, mechanically assisted gait, joint position biofeedback, virtual reality training, ankle-foot orthoses, and daily living activities. Analysis of qualitative data will be achieved by way of thematic analysis involving various stages. Once transcribed verbatim, it will be read and re-read to allow the researchers to gain meaning from the verbatim. Data will then be broken down into discrete parts that will represent segments of raw data. These segments will comprise words, phrases, or large blocks of data that will be abstracted under conceptual headings. Coding will then be done for similarities and differences in the data that will involve continually comparing segments and concepts with new data, which in turn will culminate into new concepts. According to [22], this process is known as "open" coding. Data will be coded by looking closely at what participants will described themselves as performing, feeling, and being. Further, the researchers will identify conditions that will have shaped participants' experiences and then captured different and/or similar contexts that could add meaning and variation to categories that will have emerged in the data. Axial coding of data will then be done by making tentative propositions about the relationships between emerging categories and about how variation in context might shape participants' experiences as proposed in [22].

The final coding phase will be the selective coding phase and this will entail identification of a core category that incorporates other categories or supersedes them in explanatory importance (ibid). During selective coding, an integrative diagram will be developed in the initial stages and be refined in subsequent stages to provide a graphic description of the substantive theory and illustrate the relationships between concepts and categories including the core category. Or- 
ganization of data will be done using NVivo version 13 software, were as analysis will be done manually.

\subsubsection{Plan for Utilization of Data}

It is hoped that information obtained from this study will be used as a basis for recommending streamlined role of the Nurse and Physiotherapist in both acute care and rehabilitation. The information can also be used as a basis for developing teaching materials for nursing and physiotherapy students, staff and strokes patients with a potential for improved care and compliance to treatment respectively. The information will further be used as a basis to advocate for set up of acute-inpatients stroke patients' units and ideal community rehabilitation centres for stroke survivors which can be used as model for acute stroke care and rehabilitation.

\subsubsection{Plan for Dissemination}

Information from this study will be disseminated as follows:

1) Presentations during clinical meetings at the UTH-Adult Hospital;

2) Presentations during research seminars, and or journal clubs at School of Nursing Sciences and School of Health Sciences at the University of Zambia Ridgeway Campus;

3) Presentations at National Health Research Authority of Zambia Conferences;

4) Publication in peer reviewed journals.

\section{Discussion}

Globally, CVAs rank the second leading cause of death and the third leading cause of disability [1]. The 2016 Global Burden of Disease report indicated that the global prevalence of stroke stood at 80 million [1]. Studies have reported that the contributing factors to stroke include: hypertension, diabetes, obesity, low physical activity levels, elevated lipids, unhealthy diet, and smoking [23] [24]. A study conducted by [25] projected that about $80 \%$ of all stroke cases will occur in low- and middle-income countries of the world by the year 2030. Of the prevailing cardiovascular diseases in Africa, stroke was the fourth leading cause of all deaths in 2015 [26].

With regards to disability, stroke remains a leading cause of long-term disability in the United States at a cost of $\$ 38$ billion per year [27]. The Canadian report further states that about 650,000 persons survive a new stroke yearly and 7 million Americans live with the complications of stroke which mostly lead to disability. The prevalence of stroke is likely to increase in the future due to the aging population which is equally increasing due to improved health care services throughout the world [1] including in low- and medium-income countries [28] such as Zambia.

In Zambia which is a middle-income country, cardiovascular disorders are the most prevalent Non-Communicable Diseases (NCDs), with an estimated preva- 
lence of over 50,000 cases at the primary health care level in 2008 and 2009 [29]. In 2016, it was reported that NCDs caused $23 \%$ of all deaths in the country, with nearly one in five people dying prematurely from these conditions [30]. In another cross-section study that was conducted at UTH to describe the clinical characteristics and outcomes of stroke patients admitted to this Hospital between June to December 2010, a total of 250 stroke patients were included in the study, out of which $65 \%$ had ischemic stroke and $35 \%$ hemorrhagic stroke mainly caused by hypertension [31].

Due to the diversity of impairment that results from stroke, care and rehabilitation for stroke survivors require a multidisciplinary approach. There is unequivocal evidence of improved outcomes when patients are treated in a stroke unit by multi-disciplinary teams [6]. [8] reported that in an ideal situation, a multi-disciplinary team takes care of a Stroke patient and the team comprises of the following professionals: doctors, nurses, physiotherapists, occupational therapists, dieticians, speech and language therapists, neuropsychologists, stroke liaison sister/nurse, family and care support worker, stroke specialist, nurse and care managers. Each of these professionals has a specific responsibility towards holistic and comprehensive care and rehabilitation of stroke patients.

Nurses and physiotherapists are reported to be the largest professional workforce in the rehabilitation setting and working with stroke patients [8]. The role of nurses and physiotherapists in the management of patients with CVA is therefore very critical. This is because nurses are with the patients for 24 hours and conduct most bedside monitoring [32]. Nurses are the largest of the health care professional groups, spend the most direct time with patients; thus, their role in the prognosis of the patient's condition is critical [33]. Nurses play a pivotal role in the ongoing monitoring and treatment of patients with stroke in the initial phase following admission to the hospital [34]. Specific nursing roles include measuring vital signs and the level of consciousness, assessing reflexes including the swallow reflex which determines the mode of feeding, assessing mobility and assisting, turning, the patients, and meeting the hygienic need in patients with severe stroke. In addition, nurses coordinate and accompany the patient's journey of care, liaising with other specialties, facilitating rehabilitation, discharge and follow-up planning. Therefore, the efficacy of treatment for clients with stroke is highly dependent on the nurses' ability to manage the disease thereby preventing complications such as pneumonia, pressure sores, seizures and incontinence. [35] reported that highly specialized nursing input is of paramount importance in achieving optimum patient outcomes.

Regarding the role of the physiotherapist, the Canadian Society of Physiotherapy [36], reported that physiotherapy for stroke survivors is an integral part of the rehabilitation treatment plan. As members of stroke teams, physiotherapists address physical function issues and prevention of subsequent strokes, which are key determinants of Quality of Life (QoL). Additionally, the Canadian Society of Physiotherapy [36], asserts that physiotherapists provide rehabilitation services 
which supports the individual's ability to live independently through targeted interventions aimed at improving balance, strength, coordination and function. Physiotherapy, therefore, is a key component in the continuum of care by providing support for the transition from hospital to home [37]. In the Zambia context, physiotherapists are the main rehabilitation specialists for stroke patients as the country has no Speech therapists and Occupational therapists.

In Zambia, there are several challenges that impact both acute care and rehabilitation of stroke patients. For acute hospital care, despite significant evidence of the success of organized acute stroke care units, such services are not available in most developing countries such as Zambia where stroke survivors are just managed in general wards and general outpatient departments [5]. On the other hand, among the several challenges faced in the rehabilitation of stroke survivors are the inadequate human resources for outreach programmes, skilled community workers, resources for post-illness care, and partnerships [38].

Despite Stroke being reported to be the second most common cause of death globally [1], accurate data on the role of different members of the multidisciplinary care team is mostly from studies conducted in high-income countries but scarce in most low and middle-income countries include Zambia and other Southern Africa countries [39]. Accurate prevalence data, as well as evidence-base data of care and rehabilitation services being provided to stroke patients, is equally scare in most low and middle-income countries [38] [40]. Similarly, in Zambia, according to [41], patients receive very little in-patient rehabilitation and are discharged at an acute stage. It is for the foregoing reasons that the proposed study seeks to investigate the role of the Nurse and the Physiotherapist in stroke care and rehabilitation from the Zambian context.

\subsection{Conclusion}

From the Zambia Context, there is limited information regarding the specific roles of nurses and physiotherapists in stroke management. The scarcity of such information continues to persist despite research evidence of improved outcomes when stroke patients are treated by a multi-disciplinary team. It is therefore hoped that information obtained from this study will be used as a basis for recommending streamlined role of the nurse and physiotherapist in both acute care and rehabilitation. The information can also act as motivation for other scholars to conduct related studies using more large and representative samples.

\subsection{Ethical Approval of the Project}

Ethical approval will be obtained from the University of Zambia, Biomedical Research Ethics Committee (UNZABREC). Permission to conduct the study will also be sought from the National Health Research Authority, and from the UTHAdult Hospital. Participants by with an information sheet that will outline the nature and purpose of the study, the data collection process, as well as their rights. This will be done to enable participants to make an informed and volun- 
tary decision whether to participate in the study or not. Participants will also be made cognizant of their right to withdraw from the study at any given time without having to explain doing so. Confidentiality and anonymity will be assured and maintained at all times as participants' names will not be used on any of the questionnaires, field notes or audio recordings. Furthermore, the questionnaires, field notes and audio recordings will be kept inaccessible to people other than the research team. In this study, the researchers take cognizance of the fact that as they get more from participants describing the care they provided in specific contexts, the risk of participant identification increases. To mitigate this risk, the researchers will mask contextualization to some extent to guard participants' identities, while still ensuring that what will be reported is verbatim, retaining the literal meaning described by the participant. In addition, the research team comprises nurses and physiotherapists, however, to reduce the influence of the research team's beliefs on the research process, the team will discuss how their beliefs can influence the research, and guard against such.

\subsection{Contributors}

PKM and MCB conceptualized the project, PKM further wrote the Background information, refined the protocol and drafted the first version of the manuscript. MMK and EMK wrote the methodology section, VMK wrote the statement of the problem and justification of the study, PDCP contributed in Proofreading, re-alignment of information for the article while FC, MS, CK and HS conducted the literature review.

\section{Acknowledgements}

The authors would like to thank the University of Zambia-Directorate of Research and Graduate Studies for giving a Seed-Funding for the initiation of this project. We would also like to appreciate the University Teaching Hospital Adult Hospital Lusaka for availing statistics related to stroke Morbidity and Mortality and care at the Hospital.

\section{Conflicts of Interest}

Authors declare no competing interest.

\section{References}

[1] World Health Organization (WHO) (2016) The Top 10 Causes of Death. http://www.who.int/mediacentre/factsheets/fs310/en/

[2] Donkor, E.S. (2018) Stroke in the 21st Century: A Snapshot of the Burden, Epidemiology, and Quality of Life. Stroke Research and Treatment, 2018, Article ID: 3238165. https://doi.org/10.1155/2018/3238165

[3] Lutz, B.J. and Green, T. (2016) Nursing's Role in Addressing Palliative Care Needs of Stroke Patients. Stroke, 47, e263-e265. https://doi.org/10.1161/STROKEAHA.116.013282

[4] Owolabi, M.O., Akarolo-Anthony, S., Akinyemi, R., Arnett, D., Gebregziabher, M., 
Jenkins, C., et al. (2015) The Burden of Stroke in Africa: A Glance at the Present and a Glimpse into the Future. Cardiovascular Journal of Africa, 26, S27-S38. https://doi.org/10.5830/cvja-2015-038

[5] Mapulanga, M., Nzala, S. and Mweemba, C. (2014) The Socio-Economic Impact of Stroke on Households in Livingstone District, Zambia: A Cross-Sectional Study. Annals of Medical and Health Science Research, 4, S123-S127.

[6] Stroke Unit Trialists' Collaboration (2007) Organised Inpatient (Stroke Unit) Care for Stroke. Cochrane Database of Systematic Reviews, No. 4, Article No. CD000197. https://doi.org/10.1002/14651858.CD000197.pub2

[7] Tibaek, S. (2018) The Role of Physiotherapy in the Rehabilitation of Stroke Patients with Lower Urinary Tract Symptoms. Journal of Physical Medicine, 1, 32-40. https://doi.org/10.36959/942/337

[8] Al-Humaid, A.K.S., Al-Temani, M.A. and Al-Asmari, N.S. (2018) Role of Physiotherapy and Nursing in Multidisciplinary Team in Stroke Rehabilitation. Journal of Advances and Scholarly Researches in Allied Education, 15, 152-159. https://doi.org/10.29070/15/57843

[9] Catangui, J.E. and Roberts, J.C. (2014) The Lived Experiences of Nurses in One Hyper-Acute Stroke Unit. British Journal of Nursing, 23, 143-148.

https://doi.org/10.12968/bjon.2014.23.3.143

[10] Clare, C.S. (2018) Role of the Nurse in Stroke Rehabilitation. Nursing Standard, 33, 59-66.

[11] Seidenberg, P., Cerwensky, K. and Brown, R.O. (2014) Epidemiology of Injuries, Outcomes, and Hospital Resource Utilisation at a Tertiary Teaching Hospital in Lusaka, Zambia. African Journal of Emergency Medicine, 4, 115-122. https://doi.org/10.1016/j.afjem.2014.01.006

[12] Morse, J. (2007) Sampling in Grounded Theory. In: Charmaz, K. and Bryant, A., Eds., The SAGE Handbook of Grounded Theory, SAGE, London, 229-244. https://doi.org/10.4135/9781848607941.n11

[13] Sbaraini, A., Carter, S.M., Evans, R.W. and Blinkhorn, A. (2011) How to Do a Grounded Theory Study: A Worked Example of a Study of Dental Practices. BMC Medical Research Methodology, 11, Article No. 128. https://doi.org/10.1186/1471-2288-11-128

[14] Burns, N. and Grove, S.K. (2005) The Practice of Nursing Research, Conduct, Critique and Utilization. Elservier, St Louis.

[15] Johnson, R.B. and Christensen, L.B. (2004) Educational Research: Quantitative, Qualitative, and Mixed Approaches. Allyn and Bacon, Boston.

[16] National Stroke Foundation (2010) Clinical Guidelines for Stroke Management. National Stroke Foundation, Melbourne.

https://extranet.who.int/ncdccs/Data/AUS D1_Clinical\%20Guidelines\%20for\%20St roke\%20Management.pdf

[17] Ministry of Health Zambia (2017) Nursing and Midwifery Protocols, 40.

[18] Baatar, U. (2013) Clinical Guideline on Stroke Rehabilitation. Management of Patients with Stroke: Rehabilitation, Prevention and Management of Complications, and Discharge Planning Mongolia.

[19] Flick, U. (2014) An Introduction to Qualitative Research. 5th Edition, SAGE, Los Angeles.

[20] Polit, D.F. and Beck, C.T. (2019) Nutritional Knowledge and Food Consumption of Adolescent Students in Junior High Schools in a Rural Community in the Eastern Region of Ghana. American Journal of Food and Nutrition, 7, 36-42.

[21] Bradshaw, C., Atkinson, S. and Doody, O. (2017) Employing a Qualitative Descrip- 
tion Approach in Health Care Research. Global Qualitative Nursing Research, 4, 5. https://doi.org/10.1177\%2F2333393617742282

[22] Foley, G. and Timonen, V. (2015) Using Grounded Theory Method to Capture and Analyze Health Care Experiences. Health Services Research, 50, 1195-210. https://doi.org/10.1111/1475-6773.12275

[23] Alharbi, A.S., Alhayan, M.S., Alnami, S.K., Traad, R.S., Aldawsari, M.A., Alharbi, A., et al. (2019) Epidemiology and Risk Factors of Stroke. Archives of Pharmacy Practice, 10, 60-66.

[24] Boehme, A.K., Esenwa, C. and Elkind, M.S. (2017) Stroke Risk Factors, Genetics, and Prevention. Circulation Research, 120, 472-495. https://doi.org/10.1161/CIRCRESAHA.116.308398

[25] Towfighi, A. and Saver, J.L. (2011) Stroke Declines from Third to Fourth Leading Cause of Death in the United States: Historical Perspective and Challenges Ahead. Stroke, 42, 2351-2355. https://doi.org/10.1161/STROKEAHA.111.621904

[26] World Health Organization (WHO) (2016) Global Health Estimates 2015: Deaths by Cause, Age, Sex, by Country and by Region, 2000-2015. World Health Organization, Geneva.

[27] Mitton, G. and Dionne, F. (2012) Valuation of Physiotherapy Services in Canada (CPA) Report Using MCDA Analysis for Determining Value of Physiotherapy Services. https://physiotherapy.ca/sites/default/files/valuePT/cpa valuept falls-en.pdf

[28] Baatiema, L., de-Graft Aikins, A., Sav, A., Mnatzaganian, G., Chan, C.K.Y. and Somerset, S. (2017) Barriers to Evidence-Based Acute Stroke Care in Ghana: A Qualitative Study on the Perspectives of Stroke Care Professionals. British Medical Journal Open, 7, Article ID: e015385. https://doi.org/10.1136/bmjopen-2016-015385

[29] Ministry of Health (2011) Towards Attainment of Health Related Millennium Development Goals and Other National Health Priorities in a Clean, Caring and Competent Environment. National Health Strategic Plan 2011 to 2015 (NHSP 2011-15) for Zambia, Lusaka.

[30] Ministry of Health (MoH) (2012) Annual Health Statistical Bulletin. Version 1, Ministry of Health, Lusaka.

[31] Atadzhanov, M. (2012) Stroke Characteristics and Outcomes of Adult Patients Admitted to the University Teaching Hospital, Lusaka, Zambia. The Open General and Internal Medicine Journal, 5, 3-8. https://doi.org/10.2174/1874076601205010003

[32] Butler, R., Monsalve, M., Thomas, G.W., Herman, T., Segre, A.M., Polgreen, P.M., et al. (2018) Estimating Time Physicians and Other Health Care Workers Spend with Patients in an Intensive Care Unit Using a Sensor Network. American Journal of Medicine, 131, 972.e9-972.e15. https://doi.org/10.1016/j.amjmed.2018.03.015

[33] Ziaee, E.S., Yousefi, H. and Golshiri, P. (2019) Nurses' Consultative Role to Health Promotion in Patients with Chronic Diseases. Journal of Education and Health Promotion, 8, Article No. 178.

[34] Koirala, K. and Shrestha, A. (2016) Evidence Based Nursing Care for an Acute Stroke: Theoretical Study. Bachelor's Thesis, Novia University of Applied Sciences.

[35] Theofanidis, D. and Gibbon, B. (2016) Nursing Interventions in Stroke Care Delivery: An Evidence-Based Clinical Review. Journal of Vascular Nursing, 34, 144-151. https://doi.org/10.1016/j.jvn.2016.07.001

[36] Canadian Physiotherapy Association (2012) Stroke: the Value of Physiotherapy. https://physiotherapy.ca/sites/default/files/valuePT/cpa valuept stroke-en.pdf

[37] Powers, W.J., Rabinstein, A.A., Ackerson, T., Adeoye, O.M., Bambakidis, N.C., Becker, K., et al. (2018) 2018 Guidelines for the Early Management of Patients with Acute 
Ischemic Stroke: A Guideline for Healthcare Professionals from the American Heart Association/American Stroke Association. Stroke, 49, e46-e110. Erratum in: Stroke, 2018, 49, e138. https://doi.org/10.1161/STR.0000000000000172

[38] Ministry of Health (MoH) (2013) Strategic Plan 2013-2016 Non-Communicable Diseases and Their Risk Factors. Version 1, Ministry of Health, Lusaka,

[39] Ntsiea, M.V. (2019) Current Stroke Rehabilitation Services and Physiotherapy Research in South Africa. South African Journal of Physiotherapy, 75, Article No. a475. https://doi.org/10.4102/sajp.v75i1.475

[40] Bertram, M.Y., Katzenellenbogen, J., Vos, T., Bradshaw, D. and Hofman, K.J. (2013) The Disability Adjusted Life Years Due to Stroke in South Africa in 2008. International Journal of Stroke, 8, 76-80.

https://doi.org/10.1111\%2Fj.1747-4949.2012.00955.x

[41] Khondowe, O., Rhoda, A. and Mpofu, R. (2007) Perceived Needs of Caregivers of Stroke Patients' Receiving Out-Patient Physiotherapy Treatment in Lusaka, Zambia. South African Journal of Physiotherapy, 63, Article NO. a122.

https://doi.org/10.4102/sajp.v63i1.122 